\title{
An optimization methodology for heterogeneous minor actinides transmutation
}

\author{
Timothée Kooyman ${ }^{*}$, Laurent Buiron, and Gérald Rimpault \\ CEA, DEN, DER, CEA Cadarache, 13108 Saint Paul lez Durance Cedex, France
}

Received: 6 July 2017 / Received in final form: 30 November 2017 / Accepted: 22 January 2018

\begin{abstract}
In the case of a closed fuel cycle, minor actinides transmutation can lead to a strong reduction in spent fuel radiotoxicity and decay heat. In the heterogeneous approach, minor actinides are loaded in dedicated targets located at the core periphery so that long-lived minor actinides undergo fission and are turned in shorterlived fission products. However, such targets require a specific design process due to high helium production in the fuel, high flux gradient at the core periphery and low power production. Additionally, the targets are generally manufactured with a high content in minor actinides in order to compensate for the low flux level at the core periphery. This leads to negative impacts on the fuel cycle in terms of neutron source and decay heat of the irradiated targets, which penalize their handling and reprocessing. In this paper, a simplified methodology for the design of targets is coupled with a method for the optimization of transmutation which takes into account both transmutation performances and fuel cycle impacts. The uncertainties and performances of this methodology are evaluated and shown to be sufficient to carry out scoping studies. An illustration is then made by considering the use of moderating material in the targets, which has a positive impact on the minor actinides consumption but a negative impact both on fuel cycle constraints (higher decay heat and neutron) and on assembly design (higher helium production and lower fuel volume fraction). It is shown that the use of moderating material is an optimal solution of the transmutation problem with regards to consumption and fuel cycle impacts, even when taking geometrical design considerations into account.
\end{abstract}

\section{Introduction}

Minor actinides transmutation is the process of removing selected nuclides (Am, Cm and $\mathrm{Np}$ ) from the waste and submitting them to a neutron flux in order to turn them into fission products. In the context of a closed fuel cycle where $\mathrm{Pu}$ is reused as fuel for fast reactors, the effective removal of minor actinides from the waste could lead to a reduction of the long-term radiotoxicity of the waste packages by up to two orders of a magnitude. Additionally, since minor actinides are mainly alpha emitters, this would lead to a reduction of the heat load of the long-lived waste packages which would have a positive impact on the size of a final deep geological repository [1].

Fast reactors are considered as candidates of choice to implement minor actinides transmutation, mainly because a fast spectrum is more efficient for such a process. Indeed, fast reactors lead to a lower production of higher actinides by neutron capture and have a lower neutron balance penalty due to the addition of minor actinides [2]. Two

\footnotetext{
* e-mail: timothee.kooyman@cea.fr
}

approaches have been discussed to implement transmutation in such reactors: the homogeneous and heterogeneous approach.

In the homogeneous approach, minor actinides are mixed directly with the fuel in quantities up to a few percent. Consequently, they experience a high level of neutron flux which increases the performances of the process. However, this has several drawbacks, the main one being that minor actinides loading leads to a hardening of the neutron spectrum, which has potentially negative impacts on the core feedback coefficients [3]. Additionally, this leads to a pollution of the entire fuel cycle with minor actinides, which are strong alpha and neutrons emitters and limits the flexibility of the transmutation process as it becomes dependent on the fuel management.

In the heterogeneous approach, minor actinides are loaded in dedicated targets usually located at the core periphery and denominated minor actinides bearing blankets (MABB). In this configuration, only a very limited perturbation of the core neutron spectrum can be observed. However, as the minor actinides are loaded in a low flux zone, the performances of the process are decreased compared to the homogeneous approach [4]. To compensate for this drawback, the amount of minor actinides 
Table 1. Variation ranges of the parameters considered for pin design.

\begin{tabular}{llll}
\hline All dimensions in $\mathrm{mm}$ & Lower boundary & Upper boundary & Source \\
\hline Pin diameter & 5.8 (PFR core) & 15.8 (Superphénix blanket) & Historical review from [8] \\
Gap thickness & 0.15 & 0.5 & - \\
Cladding thickness & 0.5 & 1.0 & - \\
Wrapper flat-to flat & 180 & 220 & - \\
Expansion plenum height & Depends on the core considered. Here, between $98.9 \mathrm{~cm}$ and $168.9 \mathrm{~cm}$ for the core \\
& discussed in [9]. \\
\hline
\end{tabular}

loaded in the targets is increased along with their residence time. This leads to a high heat load of the fresh assemblies and a high decay heat and neutron source after irradiation. This has severe negative impacts on the handling and transportation of the irradiated blankets and may lead to very long cooling times before reprocessing can occur, thus increasing the total inventory of minor actinides in the entire fuel cycle.

This paper focuses on the design and optimization of a heterogeneous transmutation strategy, e.g. an analysis of the process over the entire fuel cycle and not only with regards to the irradiation step. Am being the likeliest candidate for transmutation as it is responsible for most of the mid-term radiotoxicity of the long-lived waste and as it is the main minor actinide produced by reactors using MOX fuels, only its transmutation will be considered here. In a first step, the optimization methodology built here will be discussed. In a second step, the same methodology will be reviewed and illustrated using the example of neutron spectrum modification in the blankets.

\section{Description of the optimization methodology}

\subsection{Design of minor actinides bearing blankets}

MABB exhibit various specificities detailed in [5]. The main ones are:

- an increased helium production due to alpha decay of short lived $\mathrm{Cm}$ isotopes, mainly ${ }^{242} \mathrm{Cm}$, which increases the pin pressurization and thus may lead to cladding overpressure towards the end of irradiation;

- a lower power production and power density due to the absence of fissile elements in the fuel at the beginning of irradiation;

- an important increase in the power of the blankets (up to three times higher) during irradiation as breeding occurs in the blankets over its lifetime, thus leading to a production of ${ }^{239} \mathrm{Pu}$ and ${ }^{242 \mathrm{~m}} \mathrm{Am}$. This may result in overcooling of the assemblies at the beginning of irradiation, however this issue was not considered here; - a high flux gradient at the core periphery.

The target pre-design algorithm described in [5] was used to compute an acceptable assembly design knowing the expected Am concentration in the blankets. The main hypothesis of this algorithm is that total release of the gaseous fission products and helium produced during irradiation occurs. This allows calculation of the primary strain on the cladding and thus the evaluation of the feasibility of the design. The algorithm also computes the fuel centerline temperature and Am content in $\mathrm{U}_{x} \mathrm{Am}_{1-x} \mathrm{O}_{2}$ compound, which can also be used as limiting criterion. The cladding maximal acceptable strain was compared to the one of various oxide dispersed steels. Considering the lack of available data on the resistance of these kinds of steels and their behavior under irradiation, an arbitrary value of $550 \mathrm{MPa}$ was considered for the limiting primary constraint of the cladding here based on the results obtained for two steels evaluated in [6] and previous results obtained at CEA. Such kind of steels are expected to be available in the near-future and exhibit better irradiation properties than current austenitic steels such as HT9 or AIM1 [7].

Based on the blankets assemblies used in past reactors in order to ensure technological feasibility of the results obtained, as set of boundaries for the assembly design parameters was chosen and is given in Table 1. A maximal Am content of 20 at.\% was considered throughout the study to account for potential limitations due to manufacturing of Am bearing fuels. A spacing wire of $1 \mathrm{~mm}$ was considered regardless of the pin diameter. Knowing the thermal conductivity of the fuel, helium production, cladding resistance and power level, it is thus possible to design a complete assembly within the boundaries of Table 1 and with adequate pin pressurization and fuel centerline temperature.

\subsection{Description of the optimization process}

Two objectives were pursued with this optimization process: the maximization of the Am consumption during irradiation and the minimization of the impacts on the fuel cycle. The consumption was evaluated as the Am content difference for an assembly between the beginning and end of irradiation.

Regarding the impacts on the fuel cycle constraints, it is first necessary to detail the recycling strategy considered for this study. A scenario where fuel cycle closure is achieved using only fast reactors was considered here. This is shown in Figure 1. Fresh fuel is irradiated in a fast reactor, after irradiation it is cooled and reprocessed. $\mathrm{Pu}$ and $\mathrm{U}$ are recovered to be used in standard fuel assemblies, or drivers, while Am is loaded into target assemblies located at the core periphery. Similarly, irradiated targets are allowed to cool down and are then reprocessed, with $\mathrm{Pu}$ being used for driver fuels and Am being re-irradiated. $\mathrm{Cm}$ 


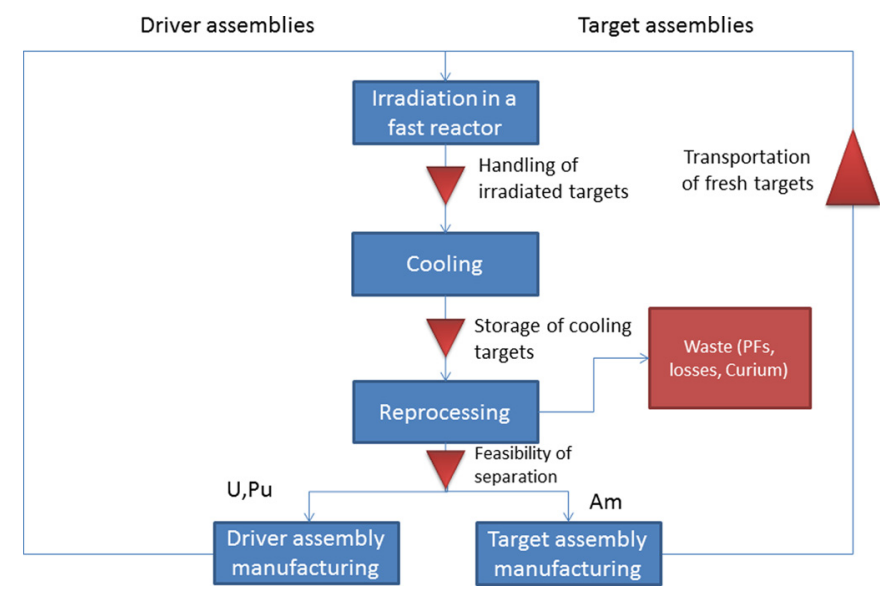

Fig. 1. Fuel cycle considered for this study.

and fission products are considered as waste and discarded during the reprocessing step. Np was not accounted for here but it can be safely assumed that it could follow the same flowsheet as $\mathrm{Pu}[10]$.

Various technological limitations can be found throughout this fuel cycle. They are shown using red arrows on the flowsheet in Figure 1. Due to their higher Am content, both fresh and irradiated targets have a higher decay heat than fuel assemblies. This complicates the transportation of fresh targets, their removal from the core after irradiation and the transportation of the targets after cooling down to their reprocessing site. If no modifications to the fuel cycle are done, the higher decay heat leads to a longer cooling time before transportation of the spent targets can be achieved. Finally, the question of the actual feasibility of the separation process remains to be addressed but this will not be treated in this study.

By considering the total inventory of Am in the fuel cycle, it is possible to take into account all those constraints in a single numerical value, which simplifies the optimization process. Indeed, for an equilibrium between the production of $\mathrm{Am}$ in the core and its consumption in the blankets, an approximation of this inventory can be written as shown in equation (1), were $m_{0}$ the initially loaded mass of Am in the blankets and $T_{x}$ the time required to accomplish the step $x$ of the fuel cycle. A minimal cooling time of 5 years was considered throughout the study.

$$
I=m_{0}\left(1+\frac{T_{\text {cooling }}+T_{\text {manufacturing }}}{T_{\text {irradiation }}}\right) .
$$

This equation represents an equilibrium situation between core production and blankets consumption. In such a situation, the total mass of Am in the fuel cycle is equal to the mass in the core initially loaded in the core, plus the mass cooling down and being reprocessed. The fraction of the loaded mass which is undergoing reprocessing depends on the ratio of the reprocessing time over the irradiation time. Higher fuel constraints lead to an increase in the cooling or manufacturing time, which increases the total inventory.
The equilibrium hypothesis between production and consumption of Am between core and blankets is valid from a neutronic point of view, as the neutron leakage of a fast reactor is generally sufficient to transmute the Am produced in the core, but may not hold true when the fuel cycle constraints are considered, as they tend to limit the amount of Am that can be loaded in a given target and thus the amount of minor actinides that can be transmuted in the blankets.

The optimization methodology of the heterogeneous transmutation strategy developed in [11] was coupled here with the assembly pre-design algorithm described above. This methodology is based on the characterization of the entire transmutation process in the blankets (transmutation performances, decay heat and neutron source evolution, flux level) based on four parameters, namely:

- the $r$-factor, which is an estimator of the neutron spectrum in the blankets. This factor is defined as the inverse of the lethargy difference between creation and absorption of a neutron. The value of the $r$-factor increases with the hardness of the spectrum, with typical values for a fast reactor being between 0.15 and 0.35 . Depending on the type and amount of moderating material considered $r$-factor as low as 0.01 can be achieved in minor actinides bearing targets with hydrogenated moderating materials as it will be shown later. It should be mentioned here that the value of the $r$ factor in subcritical medium and especially radial blankets is not physical, as a $r$-factor of 0.01 would lead to a ratio between the neutron creation and absorption energy of $2.7 \times 10^{43}$. However, this value as computed by the ECCO cell code [12] was found to be a good estimator of the neutron spectrum hardness in the blankets and was therefore used in this study. The $r$-factor thus calculated increases with the spectrum hardness;

- the Am fraction in the homogenized medium corresponding to the blanket assembly, denominated Am thereafter;

- the irradiation time $T$;

- the neutron flux $\phi$.

Artificial neural networks (ANN) with one layer of 10 hidden neurons have been trained to reproduce the output of full core calculations from the four parameters described above. These meta-models were trained on complete calculations carried out using the ERANOS code system [12] and the DARWIN depletion code [13]. They were then coupled with a genetic algorithm to obtain the set of optimal neutron spectrum and Am loading with regards to two objectives which were the amount of minor actinides consumed during irradiation and the inventory in the fuel cycle. The neutron spectrum in the blankets was tuned by modifying the volume fraction of hydrogenated material, $\mathrm{ZrH}_{2}$ in this case. It was considered the $\mathrm{ZrH}_{2}$ addition was done by displacing fuel in the assembly. The ANN presented here were trained based on a $3600 \mathrm{MW}$ homogeneous oxide core based on the design from [9]. This core will be designed as V2b thereafter.

A breakdown of the errors associated with the use of ANN is given in Table 2. It can be seen that the mean error of the meta-models is close to zero, with standard deviations around $3 \%$ for decay heat and transmutation 
Table 2. Mean error and standard deviation of the artificial neural networks used for the study of the oxide core behavior.

\begin{tabular}{|c|c|c|c|c|c|c|c|}
\hline Parameter & $\begin{array}{l}\text { Transmutation } \\
\text { rate }\end{array}$ & $\begin{array}{l}\text { Decay } \\
\text { heat @ } \\
5 \text { years }\end{array}$ & $\begin{array}{l}\text { Decay } \\
\text { heat @ } \\
10 \text { years }\end{array}$ & $\begin{array}{l}\text { Decay } \\
\text { heat @ } \\
20 \text { years }\end{array}$ & $\begin{array}{l}\text { Decay } \\
\text { heat @ } \\
50 \text { years }\end{array}$ & $\begin{array}{l}\text { Decay } \\
\text { heat @ } \\
100 \text { years }\end{array}$ & $\begin{array}{l}\text { Moderator } \\
\text { fraction for a } \\
\text { given spectrum }\end{array}$ \\
\hline Mean error $(\%)$ & 0.06 & -0.03 & 0.30 & 0.07 & 0.01 & 0.06 & 0.07 \\
\hline Standard deviation (\%) & 1.15 & 1.89 & 2.52 & 1.73 & 2.05 & 1.71 & 0.30 \\
\hline Parameter & $\begin{array}{l}\text { Neutron } \\
\text { source @ } \\
5 \text { years }\end{array}$ & $\begin{array}{l}\text { Neutron } \\
\text { source @ } \\
10 \text { years }\end{array}$ & $\begin{array}{l}\text { Neutron } \\
\text { source @ } \\
20 \text { years }\end{array}$ & $\begin{array}{l}\text { Neutron } \\
\text { source @ } \\
30 \text { years }\end{array}$ & $\begin{array}{l}\text { Neutron } \\
\text { source @ } \\
50 \text { years }\end{array}$ & $\begin{array}{l}\text { Neutron } \\
\text { source @ } \\
100 \text { years }\end{array}$ & $\begin{array}{l}\text { Helium } \\
\text { production }\end{array}$ \\
\hline Mean error $(\%)$ & 0.01 & 0.28 & 0.20 & 0.08 & 0.02 & -0.08 & -0.13 \\
\hline Standard deviation (\%) & 3.91 & 4.28 & 3.08 & 2.99 & 2.89 & 4.02 & 2.98 \\
\hline
\end{tabular}

Table 3. $Q^{2}$ estimator for the parameters of interest.

\begin{tabular}{|c|c|c|c|c|c|c|c|}
\hline Parameter & $\begin{array}{l}\text { Neutron } \\
\text { source @ } \\
5 \text { years }\end{array}$ & $\begin{array}{l}\text { Neutron } \\
\text { source @ } \\
10 \text { years }\end{array}$ & $\begin{array}{l}\text { Neutron } \\
\text { source @ } \\
20 \text { years }\end{array}$ & $\begin{array}{l}\text { Neutron } \\
\text { source @ } \\
30 \text { years }\end{array}$ & $\begin{array}{l}\text { Neutron } \\
\text { source @ } \\
50 \text { years }\end{array}$ & $\begin{array}{l}\text { Neutron } \\
\text { source @ } \\
100 \text { years }\end{array}$ & $\begin{array}{l}\text { Moderator } \\
\text { fraction for a } \\
\text { given spectrum }\end{array}$ \\
\hline$Q^{2}$ & 0.9996 & 0.9995 & 0.9998 & 0.9998 & 0.9999 & 0.9996 & 0.9997 \\
\hline Parameter & $\begin{array}{l}\text { Transmutation } \\
\text { rate }\end{array}$ & $\begin{array}{l}\text { Decay } \\
\text { heat @ } \\
5 \text { years }\end{array}$ & $\begin{array}{l}\text { Decay } \\
\text { heat @ } \\
10 \text { years }\end{array}$ & $\begin{array}{l}\text { Decay } \\
\text { heat @ } \\
20 \text { years }\end{array}$ & $\begin{array}{l}\text { Decay } \\
\text { heat @ } \\
50 \text { years }\end{array}$ & $\begin{array}{l}\text { Decay } \\
\text { heat @ } \\
100 \text { years }\end{array}$ & $\begin{array}{l}\text { Helium } \\
\text { production }\end{array}$ \\
\hline$Q^{2}$ & 0.9995 & 0.9998 & 0.9998 & 0.9998 & 0.9999 & 0.9999 & 0.9999 \\
\hline
\end{tabular}

rate. Errors for the neutron source parameter are slightly higher due to decay of ${ }^{244} \mathrm{Cm}$ during irradiation, which is the main contributor to spent fuel neutron source.

ANNs were also trained to evaluate the helium production in the blankets and thus compute the pin pressure at the end of irradiation. An additional ANN was created to calculate the amount of moderating material in the blankets required to achieve a given spectrum for a given concentration of Am in the blankets. Furthermore, it was considered that the sodium fraction in the blanket assemblies was constant and thus that loading of moderating material led to a decrease in the fuel volume fraction. This hypothesis is conservative, as it may be possible to load moderating material by decreasing the sodium volume fraction considering the low power density of the blankets. Finally, since the flux and neutron spectrum in the blankets are linked due to self-shielding effects, a last artificial neural network was built to match the flux in the blankets knowing the neutron spectrum and the core considered.

Beyond the simple evaluation of the mean and standard deviation of the neural networks outputs shown, it is possible to compute the quality of the meta-models by calculating the so-called $Q^{2}$ factor [14] which is defined below in equation (2), where $y_{i}$ is the value of the complete calculation at the point $i, \quad \tilde{y}_{i}$ the value calculated by the artificial neural network and $y$ the mean value of all the $y_{i}$. This factor is a measure of how well the meta-models reproduce the variance of the actual model.

A meta-model will be deemed acceptable if the $Q^{2}$ estimator is higher than 0.95 in this context [15]. As it is shown in Table 3 , it can be observed that all the estimators

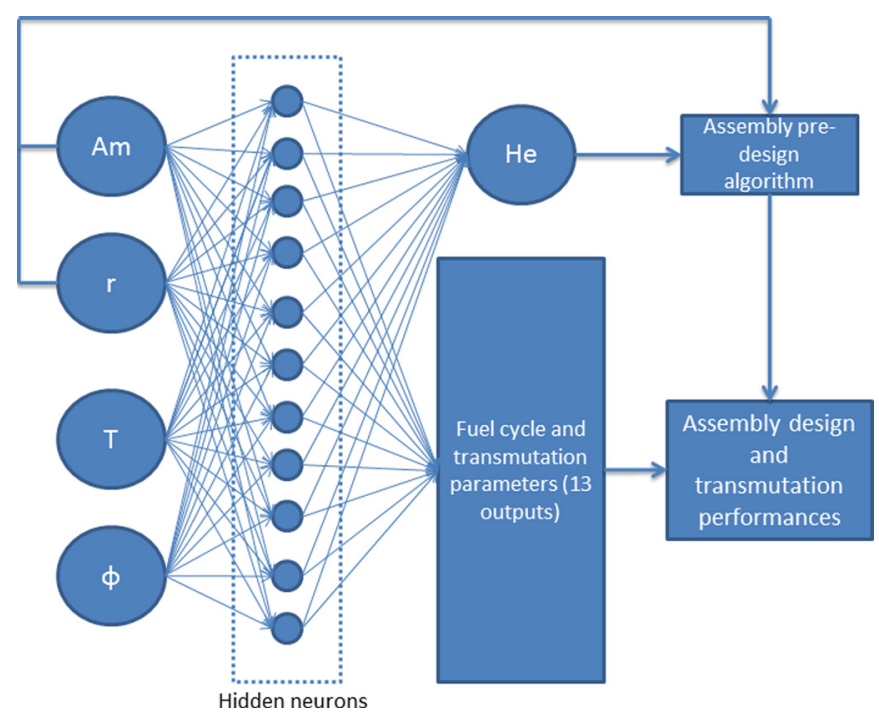

Fig. 2. Overview of the approach considered here.

studied here exhibit higher than $0.95 Q^{2}$ values, thus validating their good behavior.

$$
Q^{2}=1-\frac{\Sigma\left(y_{i}-\tilde{\tilde{y}}_{i}\right)^{2}}{\Sigma\left(\bar{y}-y_{i}\right)^{2}} .
$$

The layout of the optimization methodology is shown below in Figure 2. The initial Am concentration, irradiation time and neutron spectrum were first sampled with the neutron flux being evaluated using the ANN matching 
Table 4. Comparison of the outputs of a complete ERANOS calculation and the optimization methodology for two representative cases.

\begin{tabular}{lll}
\hline Unmoderated V2b assembly & $\begin{array}{l}r=0.073 \\
\mathrm{Am}=1.82 \mathrm{e} 21 \mathrm{at} / \mathrm{cm}^{3}\end{array}$ & $\begin{array}{l}\mathrm{Phi}=6.70 \mathrm{e} 14 \mathrm{n} / \mathrm{cm}^{2} \\
T=4100 \mathrm{EFPD}\end{array}$ \\
\hline Calculation route & ERANOS & ANN \\
Consumption per assembly (kg) & 11.22 & 11.17 \\
Decay heat @ 5 years (kW) & 8.5 & 8.4 \\
Decay heat @ 50 years (kW) & 4.1 & 4.1 \\
Assembly mass (kg) & 143.4 & 143.3 \\
Moderator fraction (vol \%) & 0 & 0 \\
\hline $\mathrm{ZrH}_{2}$ moderated V2b assembly & $r=0.0287$ & $\mathrm{Phi}=4.90 \mathrm{e} 14 \mathrm{n} / \mathrm{cm}^{2}$ \\
& $\mathrm{Am}=1.44 \mathrm{e} 21 \mathrm{at} / \mathrm{cm}^{3}$ & $T=4100 \mathrm{EFPD}$ \\
\hline Calculation route & $\mathrm{ERANOS}$ & $\mathrm{ANN}$ \\
Consumption per assembly & 11.21 & 11.24 \\
Decay heat @ 5 years & 8.9 & 9.0 \\
Decay heat @ 50 years & 4.1 & 4.2 \\
Assembly mass & 124.9 & 121.2 \\
Moderator fraction & 5 & 5.77 \\
\hline
\end{tabular}

spectrum and flux. Knowing this information, it is then possible to evaluate the required helium production and to obtain an assembly design with adequate pin pressure at the end of irradiation. Knowing the amount of fuel displaced by moderating material, the initial mass of Am loaded can be calculated. Using the corresponding ANN, the consumed mass, decay heat and neutron source at various stages of cooling can finally be computed and used as fitness estimators to carry out an effective optimization process.

The genetic algorithm available in the URANIE platform [16] was used in this work. Each case was coded using the four parameters described previously and its transmutation performances and fuel cycle impacts were evaluated in the shape of the mass consumed per assembly and the associated fuel cycle inventory. A Pareto dominance criterion [17] was used to rank the various cases obtained. A survival rate of $40 \%$ was considered here, with the remaining cases being generated by randomly selecting and optionally mutating with a $1 \%$ chance the cases from the previous generation.

Considering the specificities highlighted above, the target assemblies design was performed with the following objectives:

- maximizing the fuel volume fraction in the assembly so as to minimize the $\mathrm{Am}$ content in the $\mathrm{U}_{x} \mathrm{Am}_{1-x} \mathrm{O}_{2}$ compound. Qualitatively, this has a positive effect on the manufacturing step by reducing the specific activity of the fuel and limiting the changes in its thermodynamic behavior. In order to maximize fuel volume fraction, it is necessary to increase the pin diameter to increase the packing fraction;

- keeping the pressure inside each pin below a threshold corresponding to the maximal allowable Hoop stress on the cladding. This requires either increasing the size of the expansion volume inside the pins in order to accommodate the gaseous release inside the free space, or decreasing the pin diameter in order to limit the amount of fuel inside each pin and thus the gases production;

- keeping fuel centerline below the melting temperature of the considered fuel, e.g. $2740{ }^{\circ} \mathrm{C}$ for oxide fuel. It should be mentioned here that due to the low power in the blankets, this temperature was never reached during the optimization process;

- obtaining a neutron spectrum corresponding to the expected values by modifying the $\mathrm{ZrH}_{2}$ volume fraction in the assembly. It was considered that $\mathrm{ZrH}_{2}$ addition to the assembly was done by displacing fuel, which is a conservative hypothesis since it may be possible to replace sodium by moderating material considering the low power in the blankets.

Generally speaking, the limiting factors for assembly design were found to be the pin pressurization and the increase in the Hoop stress in the cladding.

A tentative validation of this methodology was done by comparing the results of the obtained using ANN with the results of a complete core calculation carried out using ERANOS. Two cases with similar performances are presented here, one with $\mathrm{ZrH}_{2}$ as moderating material and one without for a reference $\mathrm{V} 2 \mathrm{~b}$ assembly. The results are shown in Table 4. The optimization methodology exhibits a very good agreement with the ERANOS calculation for the unmoderated cases, with a slightly less good agreement in the moderated cases due to a higher calculated moderator fraction, however, the errors are within acceptable ranges.

\subsection{Uncertainty analysis of the meta-model approach}

The uncertainties on the Am inventory and consumption due to the use of meta-models were computed here to evaluate the accuracy of the optimization methodology. 

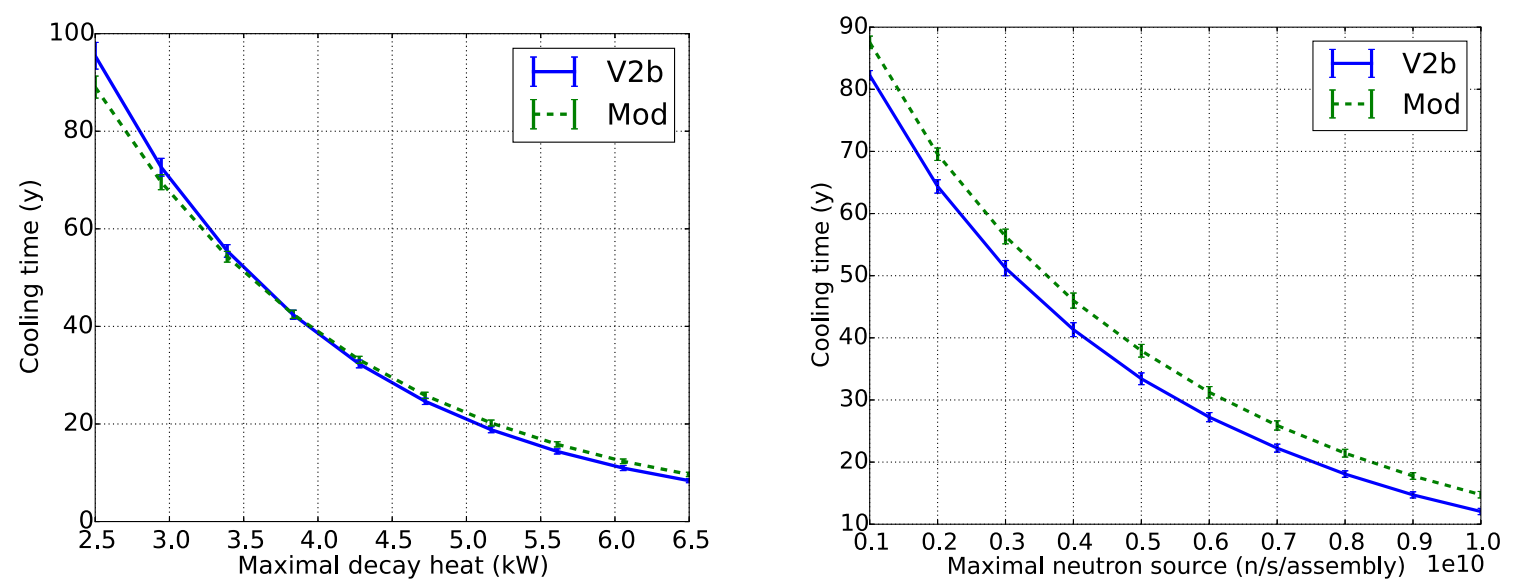

Fig. 3. Evaluation of the errors due to the meta-models approximation on the cooling time for two reference cases.

It is possible to propagate the errors on the ANN to the estimators by using a so-called "brute force" approach, where the input parameters are modified according to the uncertainties associated with the ANN and the distribution of the outputs analyzed. In a first step, the decay heat and neutron sources values were changed and the resulting cooling times distribution was fitted with a Gaussian function. The corresponding error bars can be seen in Figure 3, the two cases V2b and Mod corresponding to the cases detailed in, one without moderating material and one with $\mathrm{ZrH}_{2}$ as moderating material. For cooling times shorter than 50 years, the error on the cooling time is lower than 2 years, which was taken as the bounding value of the error on the cooling time.

The error on the Am mass in the assembly is due to the uncertainties on the actual assembly design. Helium production in the target being the main dimensioning parameter for this design, the error on the ANN was propagated to the assembly mass using the same brute force approach. The actual value of the error depends on the acceptable design constraints due to the discrete nature of the problem. Indeed, if only pin diameter and plenum height are considered as free parameters, a small increase in the helium production may require a decrease in the pin diameter to keep the total gas production per pin constant. If this decrease is too important, it is necessary to add a new ring of pins in the assembly, which modifies the fuel volume fraction in a nonmonotonous way. On the other hand, if gap and cladding thicknesses along with assembly flat-to-flat can be modified, the fuel volume variation is smoother, as it can be seen below in Figure 4. In this case, the assembly mass is obtained with a one-sigma uncertainty of $0.8 \mathrm{~kg}$. This value can be translated into an uncertainty on the Am concentration in the pins of $0.4 \%$. Considering the wider possible variations of the loaded mass, which depend on the set of constraints, this value was arbitrarily raised to $2 \%$.

The optimization process was then carried out using a $2 \%$ dispersion around the values corresponding to a V2b core. The dispersions of the inventory and fuel cycle are shown in Figure 5. In this case, the Am mass consumption

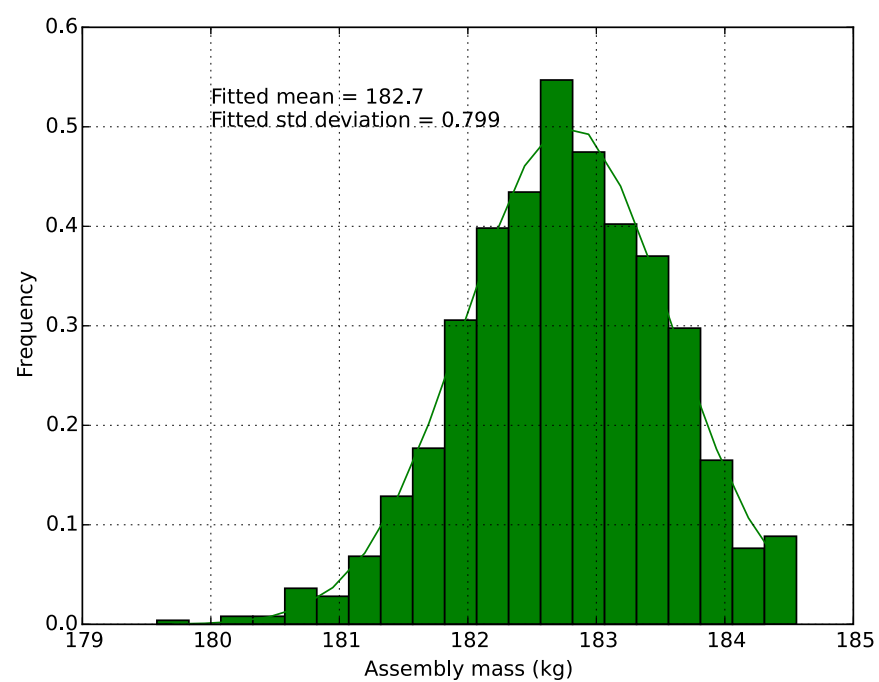

Fig. 4. Dispersion of the assembly mass due to the uncertainties on the helium production.

is obtained with a one-sigma dispersion corresponding to $2.1 \%$ of the mean consumption, while the inventory is evaluated with a $4.1 \%$ error.

A crude approximation of the total uncertainty on the inventory and fuel cycle can also be obtained using equations (3) and (4) below which neglect the correlations between the various uncertainties sources. $T$ represents the cooling time, $m$ the loaded mass in the assembly and $\tau$ the transmutation rate. It is also clear that the value of the uncertainty depends on the constraints sets on the optimization process, as the cooling time depends on the limiting value and decay heat. It can be estimated that, all sources taken into account, the uncertainty on the consumption is $3 \%$ and the uncertainty on the inventory is close to $5 \%$, and decreases with the cooling time. This rather simple analysis is consistent with the standard deviation obtained in Figure 5. The main contributor to the uncertainty on the inventory is the high relative uncertainty on the cooling time for short cooling periods. Considering the various approximations used in this methodology and its intended use for scoping studies, 

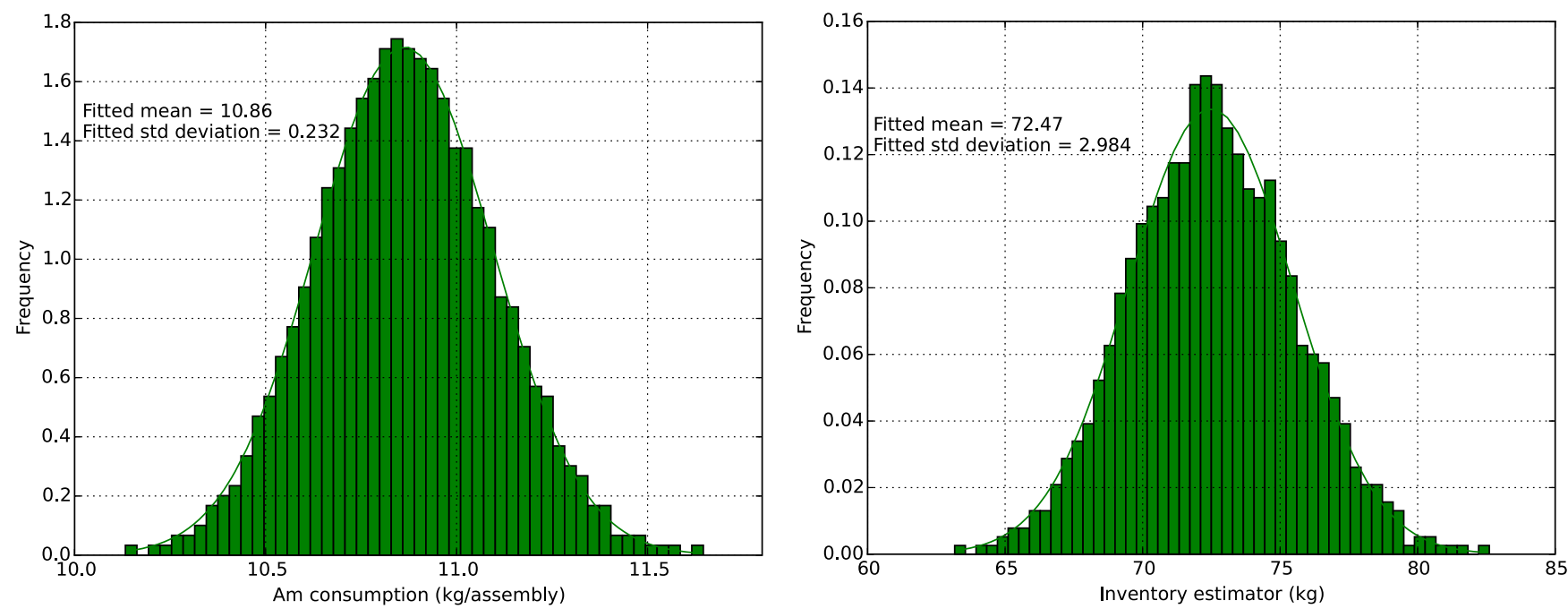

Fig. 5. Dispersion of the Am inventory and consumption per assembly due to the uncertainty on helium production.

these uncertainties estimations are considered acceptable.

$$
\begin{gathered}
\frac{\delta I}{I}=\sqrt{\left(\frac{\delta \tau}{\tau}\right)^{2}+\left(\frac{\delta m}{m}\right)^{2}+\left(\frac{\delta T}{T}\right)^{2}}, \\
\frac{\delta C}{C}=\sqrt{\left(\frac{\delta \tau}{\tau}\right)^{2}+\left(\frac{\delta m}{m}\right)^{2}} .
\end{gathered}
$$

\section{Analysis of an example: the case of the moderated approach}

Due to their location at the core periphery, the flux level experienced by MABB is lower than at the core center, which decreases the transmutation performances. To compensate for this lower flux level, it has been proposed to add moderating material, such as $\mathrm{MgO}$, Beryllium or $\mathrm{ZrH}_{2}$, in the blankets to increase the transmutation rate in the blankets region [18]. However, this approach brings the drawback of increasing the production of $\mathrm{Cm}$ and ${ }^{238} \mathrm{Pu}$ due to the increase in the capture cross sections. Consequently, moderated blankets exhibit a higher decay heat and neutron source level compared to their standard counterpart.

As an illustration of this optimization methodology, the interest of moderated blankets will be analyzed with regards to Am consumption and fuel cycle impacts while taking into account assembly design considerations. A 3600 MW oxide core was considered here as described in [19]. The irradiation time was set at 4100 EFPD, which amounts to 142.7 TWhe produced during the irradiation of the blankets. Oxide blankets with $\mathrm{ZrH}_{2}$ as moderating material were considered.

\subsection{Qualitative analysis}

At first stage, the Am inventory was calculated by considering a cooling down to $7.5 \mathrm{~kW}$ per assembly taken from [20] and only an adaptation of the pellet diameter was allowed in terms of geometrical design. The Am concentration in the MABB medium and the neutron spectrum were first considered as input parameter with a constant irradiation time of 4100 EFPD. The unmoderated V2b case described in Table 4 was used as a comparison point.

For a better visualization of the results of the optimization, the outcomes are plotted using a 2d-map with the $r$-factor on one axis and the Am initial mass per assembly on the other one. The values of the relevant estimators are then plotted using contour plot and adequate color maps.

The color map on the left of Figure 6 corresponds to an estimator of the Am inventory expressed in terms of $\mathrm{kg}$ of Am in the cycle per blanket assembly. The color map on the right represents the evolution of the Am consumption for one assembly over the irradiation time considered, expressed in $\mathrm{kg}$.

The green points correspond to cases which are not physically feasible, e.g. for which the combination $(r, \mathrm{Am})$ is not achievable, either because the neutron spectrum is too energetic for the amount of minor actinides loaded (upper-left part), or because it is not possible to achieve such a spectrum with a limited amount of $\mathrm{ZrH}_{2}$ (lower-right corner).

The red points correspond to cases which are not feasible by design, mainly here because the helium production in the pins is too important for the cladding resistance and will lead to a clad rupture at the end of irradiation. The inverted black triangles correspond to cases for which the Am content in the $\mathrm{U}_{x} \mathrm{Am}_{1-x} \mathrm{O}_{2}$ compound is higher than a limit set here at $20 \%$. Indeed, when the Am concentration in the blanket medium increases, so does the associated helium production. To accommodate this higher gas production, it is then necessary to decrease the pellet diameter. Indeed, this decreases the amount of fuel per pin and thus the gas production per pin. However, this decreases the fuel volume fraction in the assembly. To keep the Am concentration constant, the Am content in the fuel must then be raised, up until it reaches the limit of 20 at.\%. 


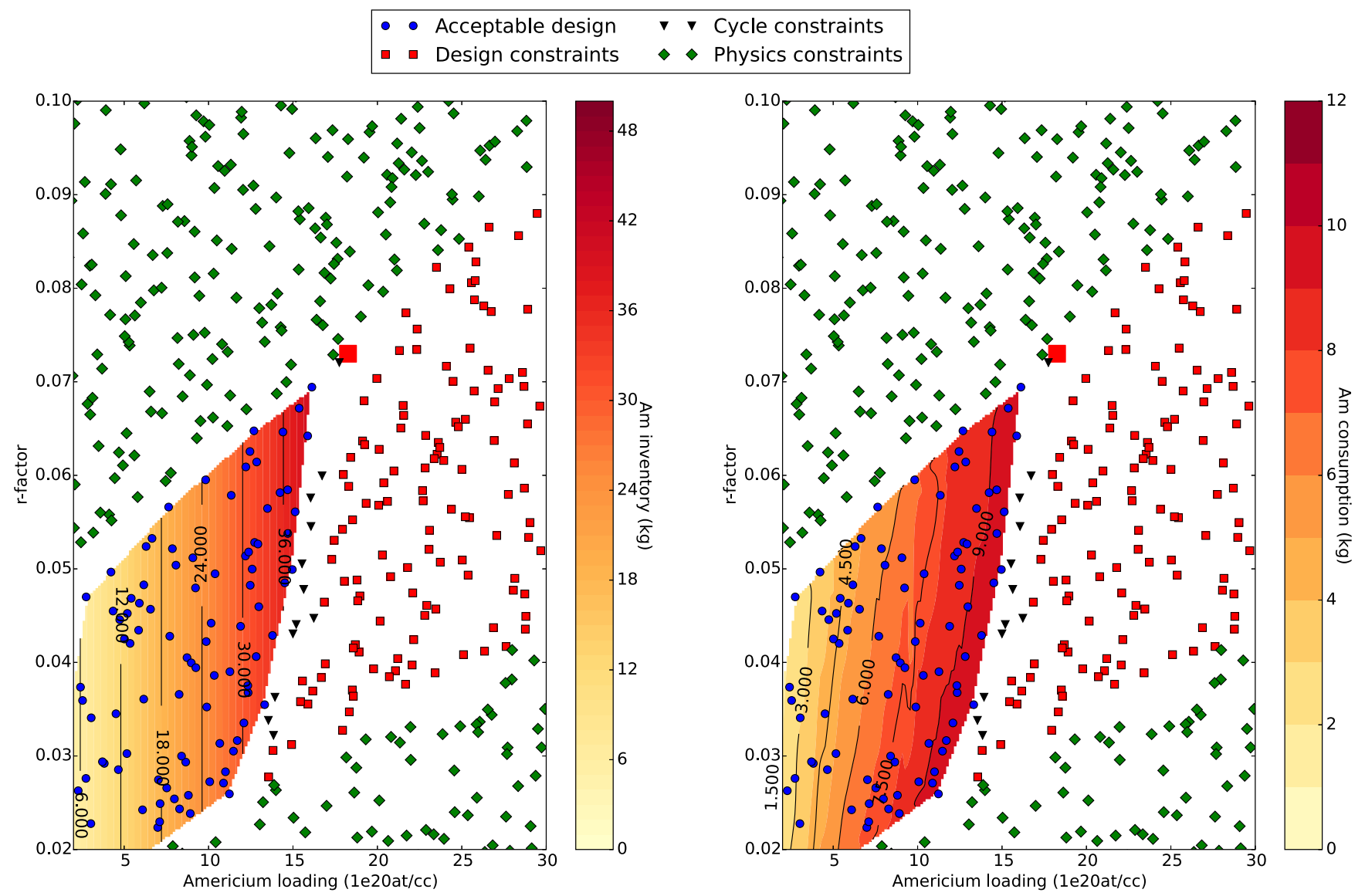

Fig. 6. Am inventory and consumption for one blanket assembly for a $3600 \mathrm{MW}$ core with a $7.5 \mathrm{~kW}$ cooling limit pellet diameter optimization. The large red square corresponds to SFR V2b situation.

The blue points correspond to cases for which it is possible to design an assembly which can withstand the increase in internal pin pressurization with the required neutron spectrum and Am loading while exhibiting an Am content in the $\mathrm{U}_{x} \mathrm{Am}_{1-x} \mathrm{O}_{2}$ fuel lower than 20 at.\%. When $\mathrm{ZrH}_{2}$ is used, which corresponds to cases with $\mathrm{r}$ values lower than 0.04, the Am content limit is the first to be reached. Indeed, it was considered here that $\mathrm{ZrH}_{2}$ replaces fuel in the assembly. Consequently, when $\mathrm{ZrH}_{2}$ is added, it is necessary to increase the $\mathrm{Am}$ content in the remaining fuel to maintain a constant Am concentration in the assembly.

Considering the vertical contour lines on the inventory plot, it can be observed that the inventory dependency on the neutron spectrum is inexistent. Indeed, with a decay heat limit set at $7.5 \mathrm{~kW}$ and the design limitations due to pin pressurization, it is not possible to obtain an assembly with a decay heat higher than this limit. Consequently, the cooling time is limited to its minimal value of 5 years and the inventory estimator only depends on the loaded mass.

On the other hand, the consumption per assembly decreases with the spectral hardening, as the total absorption rate decreases. It was also found here that the point $(\mathrm{r}, \mathrm{Am})$ corresponding to SFR V2b (larger red square) is classified as not feasible by design. This result is in good agreement with previous assembly design studies [5], which have shown that it was not possible to design an assembly with $20 \%$ Am content and 40 vol. $\%$ of fuel while adapting only the pellet radius due to the significant increase in pin pressurization.

\subsection{Impact of spectrum softening on the inventory}

To illustrate the dependency of the inventory on the neutron spectrum and on the cooling limit, the evolution of the inventory with the neutron spectrum for two Am contents and two limits were plotted in Figure 7. As shown before, for the $7.5 \mathrm{~kW}$ limit, the inventory estimator does not vary with neutron spectrum. On the other hand, for the $2.5 \mathrm{~kW}$ case, the inventory increases when the $r$-factor decreases. This is due to the higher production of ${ }^{238} \mathrm{Pu}$ and ${ }^{244} \mathrm{Cm}$ caused by the softer neutron spectrum. The effect remains relatively small, with a $10 \%$ decrease in the inventory over the spectrum range considered. It is thus not very noticeable on the color maps of the next figures.

\subsection{Geometrical optimization of the assembly}

The maximal allowable height of the gas expansion plenum was increased up to $168.9 \mathrm{~cm}$, in order to access a wider domain of feasible cases (Fig. 8). The SFR V2b case now appears to be feasible but with limited further optimization margins, which is in good accordance with previous studies. Heavier assembly designs which are feasible in terms of pin 


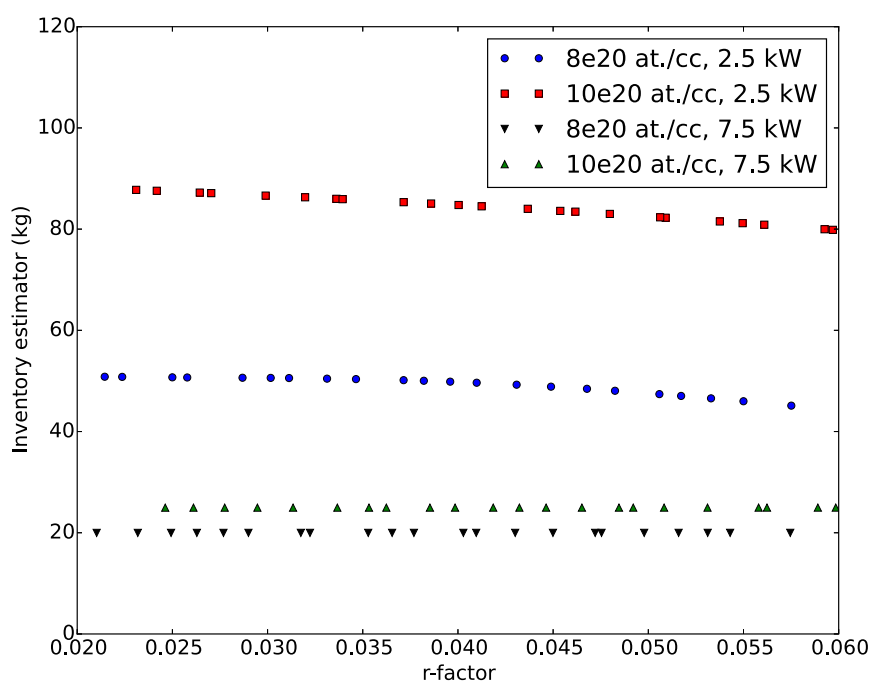

Fig. 7. Evolution of the inventory estimator with regards to the $r$-factor for various washing limits and Am loadings. Only the pellet diameter was used as an optimization parameter for the assembly design. pressurization are not feasible per se, as they would not be compatible with manufacturing constraints. Indeed, the americium content in the fuel would be higher than 20 at. $\%$. Finally, as the loaded mass increases and despite the $7.5 \mathrm{~kW}$ washing limit, the dependency of the inventory on the neutron spectrum highlighted in Figure 7 starts to appear here due to the increased production of ${ }^{244} \mathrm{Cm}$, which contributes to most of the short-term decay heat. Hence, the rightmost contour lines of the inventory plot are tilted to the right. One significant point to be observed here is that the main limiting constraint is not the assembly design but the americium content in the fuel.

Finally, all the parameters of the model shown in were used as input variables and the optimal assembly which can be obtained using a pin bundle design with 20 at.\% of Americium was computed. This is done below in Figure 9. Consumption in excess of $15 \mathrm{~kg}$ per assembly, which is equivalent to $8.8 \mathrm{~kg} / \mathrm{TWhe}$ can be reached here, with the americium content being the main limiting factor. The optimized assemblies obtained by the optimization process are characterized by high pellets diameter with thick cladding and high expansion plenum to accommodate pin pressurization, as found in [5].

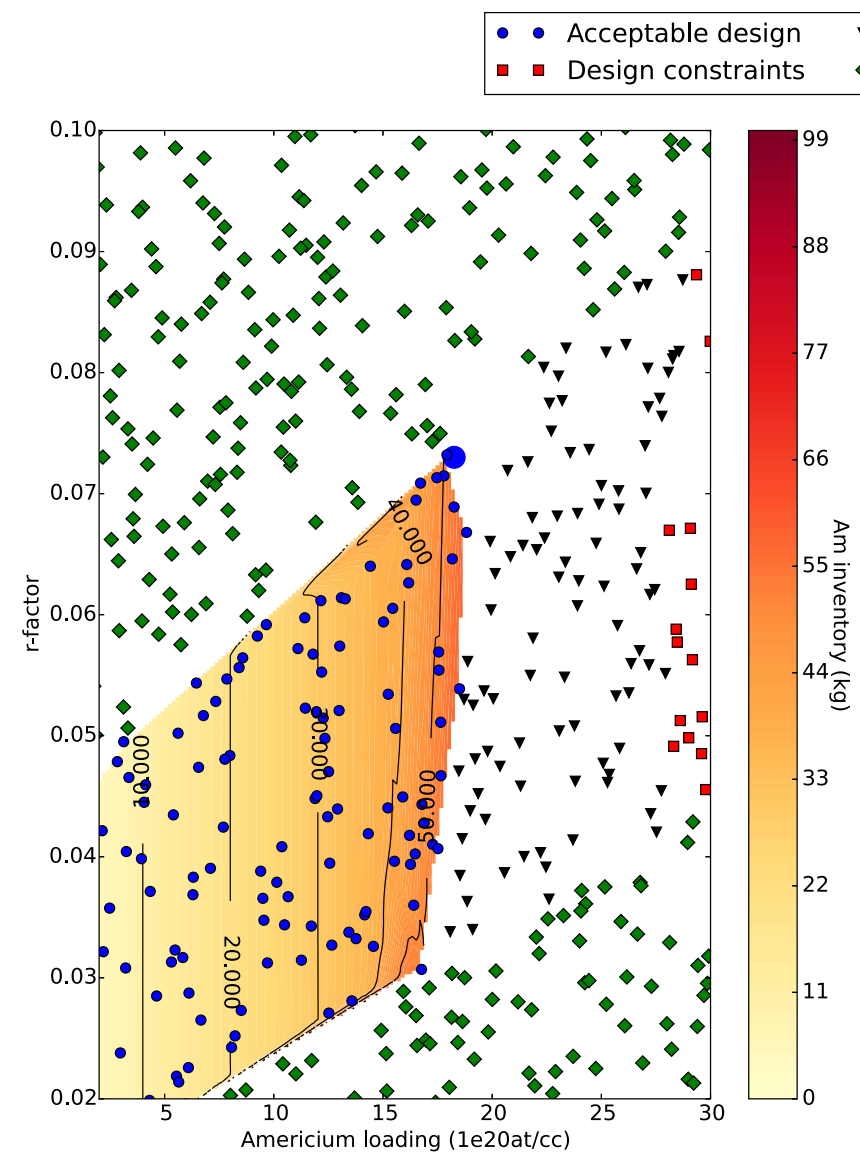

Cycle constraints

$\checkmark$ Physics constraints

Fig. 8. Am inventory and consumption for one blanket assembly for a $3600 \mathrm{MW}$ core with a $7.5 \mathrm{~kW}$ cooling limit pellet diameter and plenum height optimization. The larger blue circle corresponds to SFR V2b situation. 


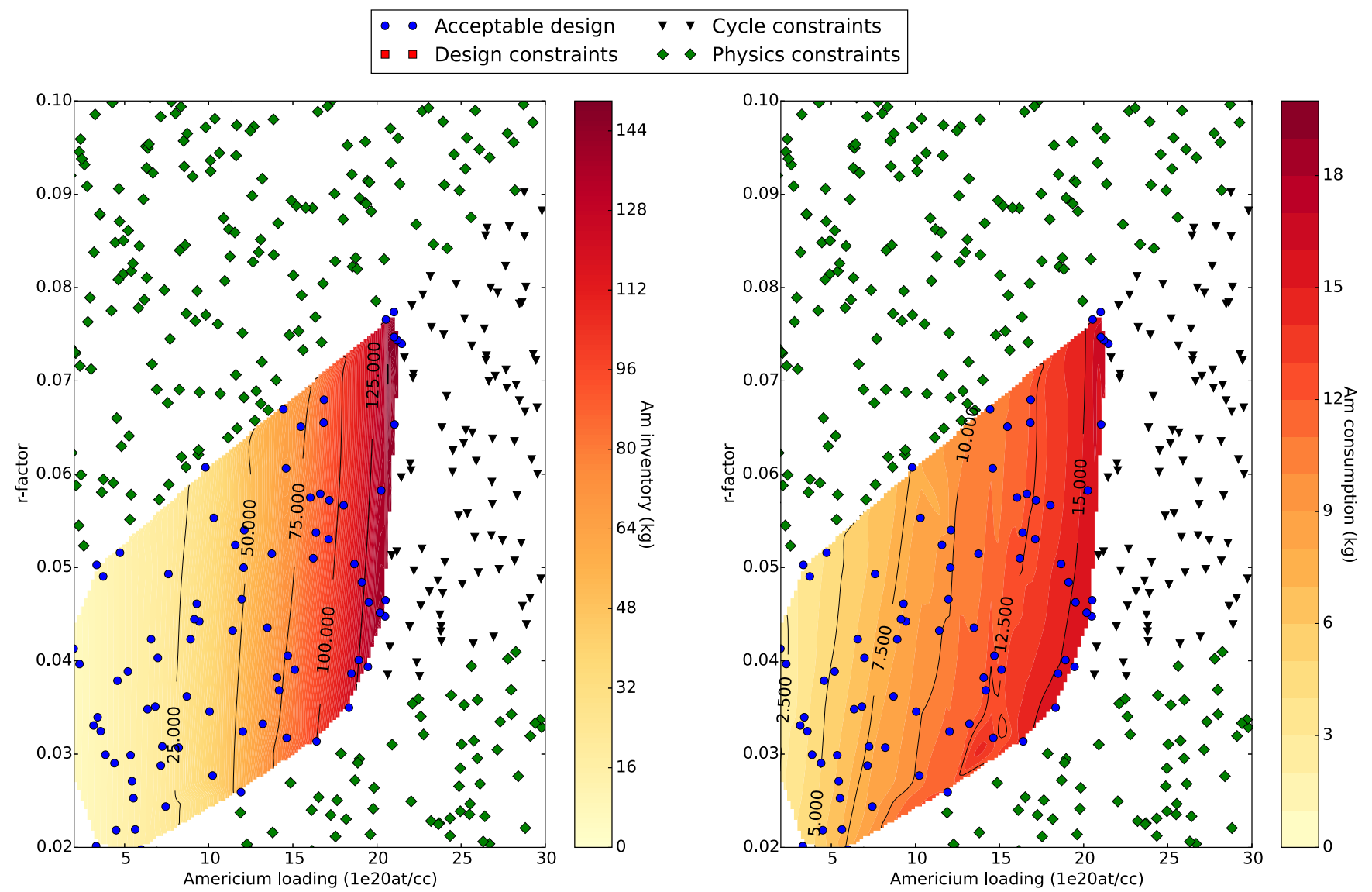

Fig. 9. Americium inventory and consumption for one blanket assembly for a $3600 \mathrm{MW}$ th oxide core conditions. A $7.5 \mathrm{~kW}$ decay heat limit was considered here. All the parameters of the assembly design model were used as free parameters. Zirconium hydride was used as moderating material.

\subsection{Complete optimization with a genetic algorithm}

It can be qualitatively on Figure 9 observed there that the cases with a heavily moderated spectrum yield the highest consumption with relatively limited inventories. A more classical optimization based on the use of a genetic algorithm was used to conclude quantitatively.

The results of the genetic algorithm optimization are depicted in Figure 10 for two cases where two limits for sodium washing were considered: 2.5 and $7.5 \mathrm{~kW}$. The left plot represents the entire set of optimal cases with regards to the Am consumption on the $x$-axis and the fuel cycle inventory on the $y$-axis. The right plot represents the same optimal cases but in the parameters space, with the $r$-factor on the $x$-axis and the Am concentration in the blankets on the $y$-axis. A case is considered optimal when it is not possible to achieve a simultaneous gain in both the consumption and the inventory by modifying the input parameters.

The assemblies were optimized to maximize the fuel volume fraction by modifying the expansion plenum height and the pellet diameter. The irradiation time was also used as an optimization parameter ranging from 2000 to 6000 EFPD. However, all the optimal cases exhibited a
6000 EFPD irradiation time as it maximizes the neutron fluence and thus the Am transmutation rate. Consequently, the time parameter was not displayed in Figure 10.

The optimal cases are located in the low $r$-factor zone of the (r, Am) phase space. The results which were found in [11] regarding the interest of moderation for heterogeneous minor actinides transmutation are thus confirmed here, even when blanket design parameters are factor in. For very low Am consumption rates, the inventory behaves similarly for the two limits, as the spent fuel decay heat is lower than $2.5 \mathrm{~kW}$. Thus, the same cooling time of 5 years is applied in both cases.

However, above around $6 \mathrm{~kg}$ per assembly $(2.35 \mathrm{~kg} / \mathrm{TWhe})$, the curve corresponding to the $2.5 \mathrm{~kW}$ limit separates from its counterpart as the irradiated blankets decay heat goes above $2.5 \mathrm{~kW}$ and its cooling time increases. Where equilibrium between core production and blanket consumption is achieved $(4.22 \mathrm{~kg} /$ TWhe in this case, or $10 \mathrm{~kg}$ per assembly for the V2b core considered here), shown as a black line on Figure 10, the inventory for the $2.5 \mathrm{~kW}$ limit case is 2.5 times higher than the one for the 7.5 case $\mathrm{kW}$ due to the longer associated cooling times. We can therefore conclude from this that the use of a "moderated" spectrum in the blankets is optimal in terms 

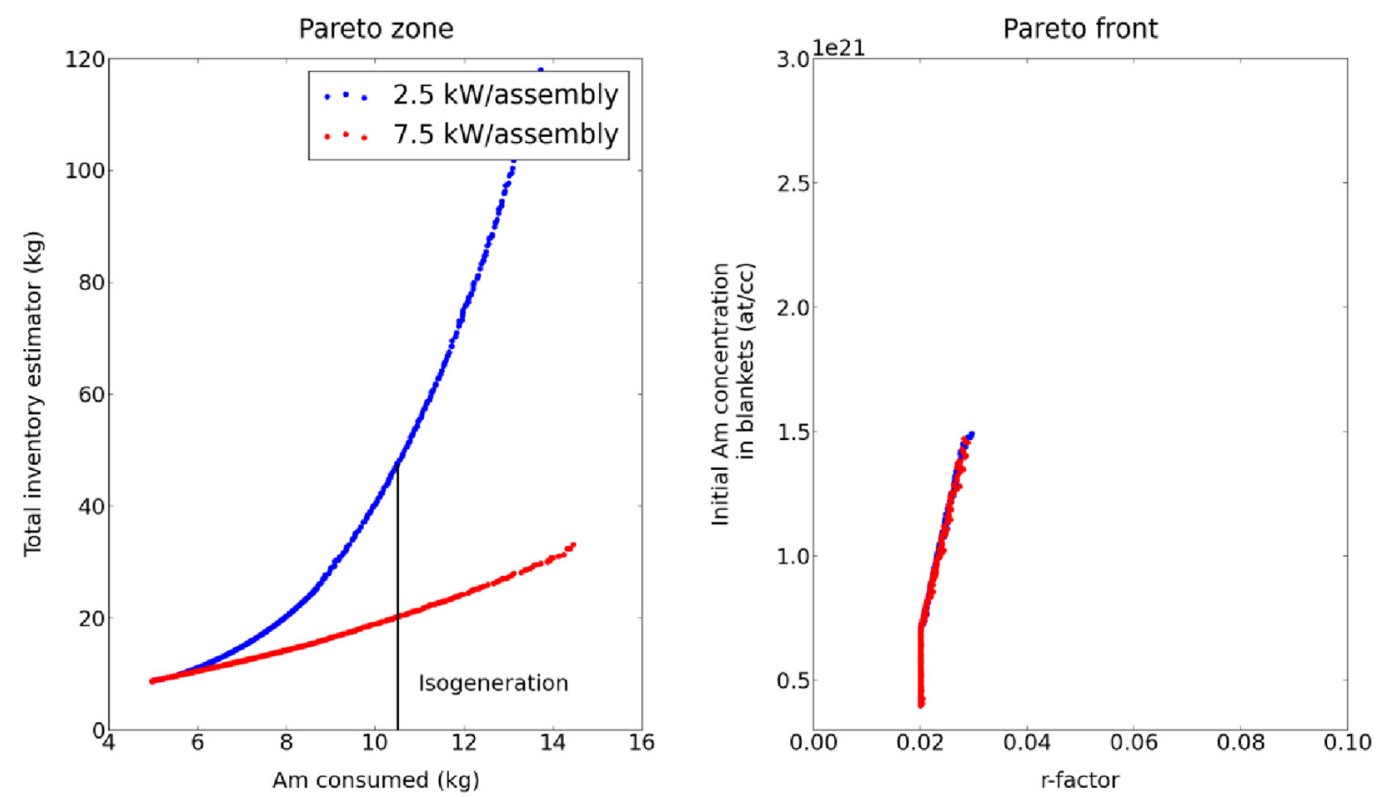

Fig. 10. Pareto front and zone with regards to consumption and Am inventory in the fuel cycle for an oxide core. The irradiation time was maximized at 6000 EFPD and the assembly design was optimized with regards to the pellet diameter and gas plenum expansion height.

of Am transmutation and fuel cycles impacts, even while taking into account assembly design (pin pressurization, moderator fraction and Am content).

\section{Conclusions}

Due to the specificities of heterogeneous minor actinides transmutation, such as high helium production or high flux gradient at the core periphery, the design of MABB differs from standard assembly design. Considering that pinpressurization and Am content in the fuel are the main limiting factor for transmutation targets design, a geometrical design methodology was used to compute the optimal target design for a given neutron spectrum and Am loading.

Then, meta-models were created to reproduce the behavior of a minor actinides bearing blanket under irradiation at the periphery of a $3600 \mathrm{MW}$ oxide core in terms of helium production, flux level, transmutation performances, decay heat and neutron source. These meta-models were coupled with the simplified assembly design routine mentioned above to obtain a tool allowing complete design and characterization of transmutation targets on a wide range of americium content, irradiation time and neutron spectrum. This tool was used to analyze the possible use of moderating material in the targets to increase transmutation performances at the expense of increased fuel cycle impacts and limited fuel volume fraction in the assembly.

It was demonstrated that the use of moderating material was optimal with regards to fuel cycle and transmutation, even while taking into account the reduction in the available fuel volume fraction due to the addition of moderating material. The increase in the transmutation rate compensates for the higher curium production. It was also shown that consumption in excess of $8.8 \mathrm{~kg}$ per TWhe could be obtained with a fully optimized geometrical assembly design characterized with wide pins, thick cladding and high gases expansion plenum, this within expected constraints linked to fuel reprocessing.

\section{References}

1. C. Chabert, D. Warin, J. Milot, A. Saturnin, A. Leudet, Impact of minor actinide transmutation options on interim storage and geological disposal, in IEMPT, 2012 (Prague)

2. NEA, Minor actinides burning in thermal reactors (NEA, Paris, 2013)

3. G. Palmiotti, M. Salvatores, M. Assawaroongreungchot, Impact of the core minor actinide content on fast reactor reactivity coefficients, J. Nucl. Sci. Technol. 48, 628 (2011)

4. NEA, Homogeneous versus heterogeneous recycling of transuranics in fast nuclear reactors (NEA, Paris, 2008)

5. T. Kooyman, L. Buiron, G. Rimpault, Optimization of minor actinide-bearing radial blankets for heterogeneous transmutation in fast reactors, Nucl. Sci. Eng. 185, 335 (2017)

6. R. Klueh, J. Shingledecker, R. Swindeman, D. Hoelzer, Oxide dispersion-strengthened steels: A comparison of some commercial and experimental alloys, J. Nucl. Mater. 341, $103(2005)$

7. M. Le Flem, P. Gavoille, Y.D. Carlan, C. Bisor, J. Seran, Advanced steel claddings for SFRS: feedback and challenges, in GEMAT Final Workshop, 2013

8. A. Waltar, A. Reynolds, Chapter 8 : Fuel pin and assembly design, in Fast Breeder Reactors (Pergamon Press, New York, 1981), pp. 251-312

9. P. Sciora, D. Blanchet, L. Buiron, B. Fontaine, M. Vanier, F. Varaine et al., Low void effect core design applied on 2400 MWth SFR reactor, in ICAPP 2011 (Nice, France) 
10. J. Tommasi, M. Delpech, J. Grouiller, A. Zaetta, Long-lived waste transmutation in reactors, Nucl. Technol. 1, 133 (1995)

11. T. Kooyman, L. Buiron, B. Valentin, G. Rimpault, F. Delage, Pre-design optimization of a target assembly for minor actinides transmutation, IEMPT 2016 (San Diego)

12. G. Rimpault, The ERANOS code and data system for fast reactor neutronic analyses, in PHYSOR, 2002 (Seoul)

13. A. Tsilanizara, C. Diop, B. Nimal, M. Detoc, L. Luneville, M. Chiron et al., DARWIN: An Evolution Code System for a Large Range, J. Nucl. Sci. Technol. Suppl. 1, 845 (2000)

14. A. Marrel, B. Iooss, E. Volkova, An efficient methodology for modeling complex computer codes with Gaussian processes, Comput. Stat. Data Anal. 52, 4731 (2008)

15. O. Fabbris, S. Dardour, P. Blaise, J.-H. Ferrasse, M. Saez, Surrogates based multi-criteria predesign methodology of sodium-cooled fast reactor cores - application to CFV-like cores, Nucl. Eng. Des. 305, 314 (2016)
16. F. Gaudier, URANIE: The CEA/DEN uncertainty and sensitivity platform, Procedia Soc. Behav. Sci. 2, 7660 (2010)

17. E. Zitzler, L. Thiele, Multiobjective evolutionary algorithms : a comparative case study and the strengh Pareto approach, IEEE Trans. Evol. Comput. 3, 257 (1999)

18. C. de Saint Jean, J. Tommasi, F. Varaine, N. Schmidt, D. Plancq, Americium and curium heterogeneous transmutation in moderated $\mathrm{S} / \mathrm{A}$ in the framework of CNE scenarios studies, in GLOBAL 2001 (Paris)

19. L. Buiron et al., Heterogeneous minor actinides transmutation on a $\mathrm{UO} 2$ blanket and on $(\mathrm{U}, \mathrm{Pu}) \mathrm{O}_{2}$ fuel in sodiumcooled fast reactor. Assessment of core performances, in Proceedings of GLOBAL, 2009 (Paris)

20. C. Chabert et al., Considerations on the industrial feasibility of scenarios with the progressive deployment of $\mathrm{Pu}$ multirecycling in SFRs in the french nuclear power fleet, in GLOBAL 2015 (Paris)

Cite this article as: Timothée Kooyman, Laurent Buiron, Gérald Rimpault, An optimization methodology for heterogeneous minor actinides transmutation, EPJ Nuclear Sci. Technol. 4, 4 (2018) 